\title{
Una visión del reimplante intencional como alternativa a la exodoncia dentaria
}

\author{
A view of intentional replantation as alternative to tooth extraction \\ Hernán Coaguila-Llerena ${ }^{1 a}$, Javier Zubiate-Meza ${ }^{1 b}$, Carlos Mendiola-Aquino ${ }^{1 b}$
}

\section{RESUMEN}

La filosofía conservadora es lo más importante en la práctica odontológica contemporánea. No sólo implica la conservación de la estructura dentaria, sino también la preservación de los tejidos circundantes. El reimplante intencional está indicado en casos donde realizar un tratamiento de conductos de manera adecuada es sumamente difícil, o cuando una cirugía apical puede verse comprometida por distintos motivos. La presente revisión bibliográfica abordará las indicaciones, contraindicaciones, principios y técnica de este procedimiento quirúrgico para-endodóntico. Asimismo, se discutirá su conveniencia como opción conservadora antes de la extracción dentaria.

\section{PALABRAS CLAVE: Endodoncia, procedimientos quirúrgicos orales, reimplante dental.}

\section{SUMMARY}

A conservative philosophy is the most important in contemporary dental practice. It involves not only the conservation of tooth structure, but also the preservation of the surrounding tissues. Intentional replantation is indicated in cases where a proper root canal is extremely difficult, or when an apical surgery may be compromised for various reasons. This literature review will address the indications, contraindications, and technical principles of this paraendodontic surgical procedure. Also, will discuss its feasibility as conservative option before tooth extraction.

KEYWORDS: Endodontics, oral surgical procedures, toothreplantation.

${ }^{1}$ Facultad de Estomatología, Universidad Peruana Cayetano Heredia. Lima, Perú.

a Cirujano Dentista.

${ }^{\mathrm{b}}$ Especialista en Endodoncia 


\section{INTRODUCCION}

La filosofía conservadora es lo más importante en la práctica odontológica, y muchas veces esto no se cumple tanto por parte del odontólogo como por parte del paciente. La conservación no sólo implica el mantenimiento de la estructura dentaria, sino también la preservación de los tejidos circundantes $(1,2)$.

En el siglo XI, Abulcasis describió el primer reporte de un reimplante y el uso de férulas en el diente reimplantado (2). Además, se recurría al reimplante intencional en el campo de batalla cuando los soldados tenían que sacrificar sus dientes para los oficiales (3). Pierre Fauchard, en 1712, reportó un reimplante intencional, quince minutos después de la extracción de la pieza dentaria de su alvéolo (2). En 1890, Scheff discutió la función del ligamento periodontal en el pronóstico de los dientes reimplantados (2). En el siglo XX también fueron practicados, pero bajo el beneficio de todas las ayudas disponibles de la ciencia moderna (2), los cuales incluyen reportes pioneros hechos por Ehriche, Loos, Faust, Schön, Heiss, y Schmidt $(2,3)$.

\section{REVISION DE LITERATURA}

El reimplante intencional consiste en extraer el diente, encontrar y corregir el defecto previo y reimplantarlo nuevamente dentro de su alvéolo $(4,5)$. Grossman en 1982 lo definió de la siguiente manera: "La remoción deliberada de un diente y su reinserción casi inmediata después de cerrar el foramen apical”(6-8).

\section{Indicaciones}

El reimplante intencional está indicado en casos donde es prácticamente imposible llevar a cabo un adecuado tratamiento o retratamiento de conductos o cuando una cirugía apical puede verse comprometida como por ejemplo: a) la ubicación del diente en la arcada, considerando el espesor del hueso que lo rodea, b) la proximidad de otras estructuras anatómicas como el nervio dentario inferior o el seno maxilar, c) la ubicación del defecto a ser corregido, d) la tolerancia del paciente a someterse a una cirugía extensa, e) la presencia de una reabsorción interna o externa (610). Otra indicación incluye pacientes que presenten trismus, es decir que no puedan realizar una apertura bucal adecuada para realizar un tratamiento endodóntico o quirúrgico (11).

El reimplante intencional tiene una ventaja sobre la cirugía apical cuando se está frente a perforaciones radiculares en la pared lingual, mesial o distal de la pieza dentaria. Incluso está indicado cuando la cirugía apical podría eliminar suficiente hueso como para causar una bolsa periodontal (10).

Cuando hay un instrumento separado que no puede ser removido, tratamientos de conductos previos groseramente sobre-obturados que interfieran con la curación, restauraciones espigo-corona que requieran, pero está contraindicada la repetición del tratamiento, o si accidentalmente hubo una avulsión del diente durante la extracción de una corona protésica la indicación más conveniente será optar por un reimplante intencional $(2,9,10)$.

Este procedimiento también está reportado como una alternativa terapéutica de las fracturas radiculares verticales y fracturas corono-radiculares complicadas (12-14). Asimismo, existen patologías como las fisuras radiculares cuyo diagnóstico en sus estadios iniciales resulta un desafío debido a las limitaciones de la mayoría de equipos de Tomografía Computarizada ConeBeam (TCCB).Entonces, si un dolor se atribuye erróneamente a una fisura radicular indetectable, por medio del reimplante intencional podría obtenerse una inspección visual directa después de la exodoncia,y si se determina que no hay fisura, el diente puede ser reimplantado $(11,15)$.

Existen situaciones poco frecuentes en las cuales el reimplante intencional es una opción de tratamiento o subsanación, como cuando debe enfrentarse la obturación de ápices en forma de "trabuco" en dientes inmaduros, y en caso de movimientos ortodónticos verticales no controlados $(9,10)$. Otra situación mucho menos frecuente para la indicación del reimplante intencional es cuando se extrajo un diente que no estaba indicado para exodoncia dentaria (10).

También ha sido reportado así como una opción de tratamiento para la sinusitis (16).

\section{Contraindicaciones}

Las contraindicaciones para realizar un reimplante intencional desde un punto de vista anatómico in- 
cluyen raíces acampanadas o divergentes, las cuales podrían fracturarse impidiendo cualquier intento de reimplante intencional $(2,4,11)$. O si hay una fractura evidente de la pieza dentaria (11).

Si existe un severo compromiso periodontal la contraindicación es categórica, pues el ligamento periodontal es esencial para el pronóstico (5).

También debe evitarse cuando la extracción de una pieza dentaria requiera una hemisección radicular o contorneado óseo, o si la pieza forma parte de una prótesis fija extensa (17).

\section{Ventajas}

La gran ventaja de realizar este procedimiento radica en que es fácil de realizar, consume menos tiempo y es menos invasivo que una cirugía apical (11). Por lo tanto, la relación costo-beneficio podría ser mayor (17).

Además, las complicaciones inherentes a una cirugía apical como son las parestesias, compromiso de seno maxilar, dolor, inflamación, o formación de bolsas periodontales se reducen considerablemente $(2,11)$.

\section{Desventajas}

Las desventajas están mayormente relacionadas a la técnica realizada, pues incluyen una posible fractura de la corona o la raíz del diente (4). También es posible que se produzca una reabsorción radicular o anquilosis posterior al procedimiento $(6,11)$.

\section{Técnica}

Previamente será necesaria una buena higiene y una terapia periodontal (11). Durante la extracción, cualquier trauma en el ligamento periodontal debe ser minimizado, es decir debe realizarse con una mínima presión, ya que cualquier daño en el cemento podría ocasionar reabsorción radicular (18). Los elevadores están contraindicados por su potencial daño al ligamento periodontal (19).

El tiempo que la pieza dentaria puede estar fuera de boca debe ser menos de 30 minutos (19). Una adecuada organización es importante para una correcta preparación y obturación retroapical al mismo tiempo que se mantiene húmedo el ligamento periodontal con una solución salina a lo largo de todo el procedimiento. Mientras más tiempo se encuentre la pieza dentaria fuera de su alvéolo más desfavorable será el pronóstico $(11,19)$.

Una medicación con antibióticos de amplio espectro como la amoxicilina está indicada a menudo, especialmente cuando hay dolor e inflamación previa al procedimiento. El paciente deberá realizar enjuagatorios con Clorhexidina al 0,12\% (15ml por 30 segundos dos veces por día), empezando un día antes del procedimiento, para disminuir el contenido bacteriano de la cavidad bucal (20).

Al momento de utilizar los fórceps, estos deben ajustarse en la corona, sin llegar a la unión cemento-adamantina. Si se coloca una goma elástica (liga) alrededor del mango del fórceps se ayudará a mantener una presión constante sobe la corona y prevendrá al clínico de una caída del diente después de la extracción $(11,20)$ (Figura 1).

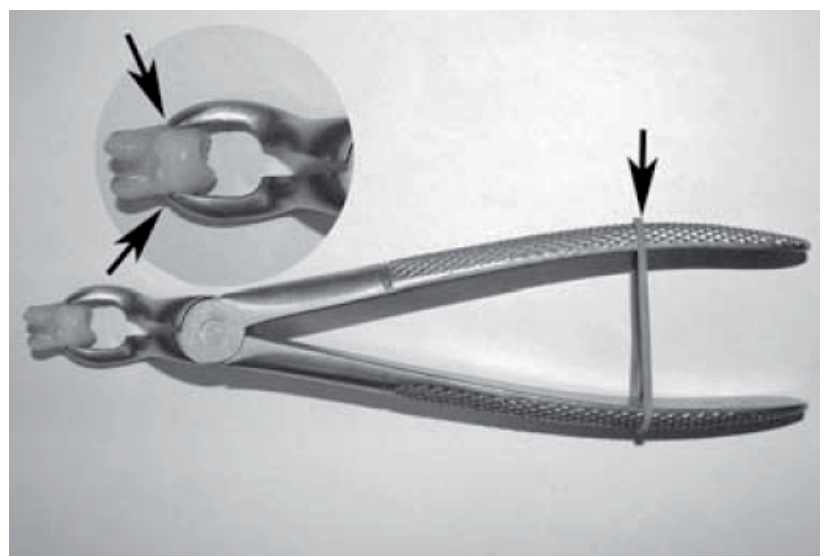

Figura 1. El uso del fórceps debe limitarse a la unión cemento-adamantina.

El uso de una goma elàstica permitirá una presión constante sobre la corona (11).

Debe evitarse la manipulación del alvéolo después de la extracción, sólo la porción apical del mismo puede ser cureteada o aspirada suavemente. Asimismo, se debe evitar tocar o desecar la superficie radicular $(10,20)$.

El ápice debe eliminarse con una turbina de alta velocidad y una irrigación copiosa con agua (Figura 2). La preparación apical debe ser plana y debe prepararse como una cavidad clase I con una fresa pequeña. Después, el ápice debe ser suavemente secado y obturado (Figura 3). Existe una variedad de cementos obturación retroapical disponibles en la literatura $(16,20)$.

Luego de colocar el diente nuevamente dentro de 


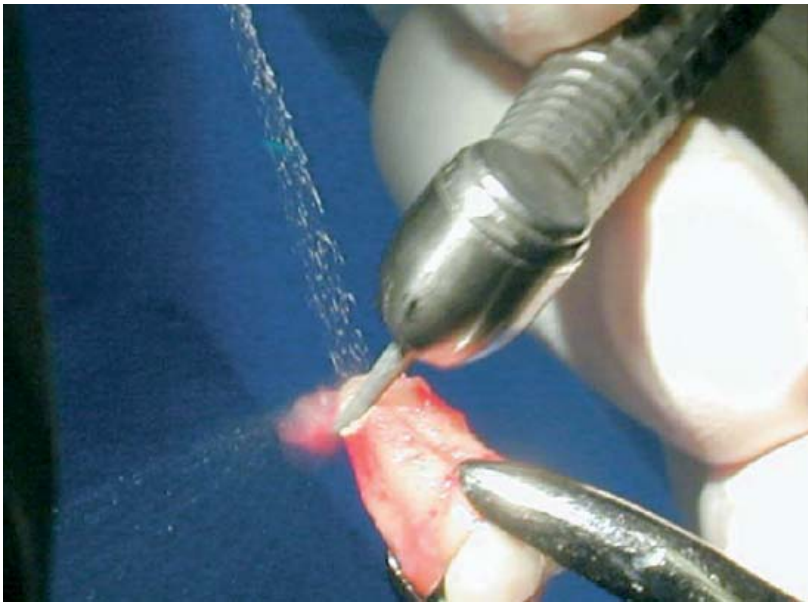

Figura 2. Apicectomía, cuya preparación debe ser plana para permitir una cavidad clase I en la obturación retroapical (17).

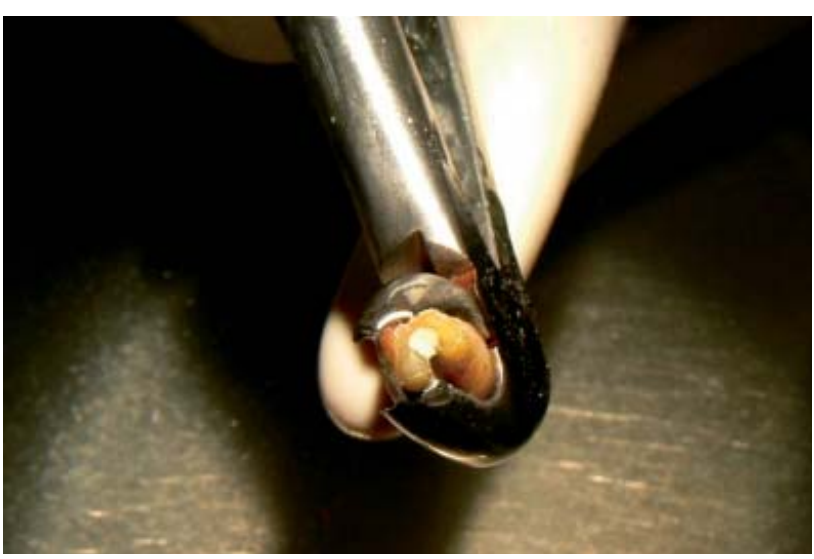

Figura 3. Obturación retroapical después de la apicectomía.

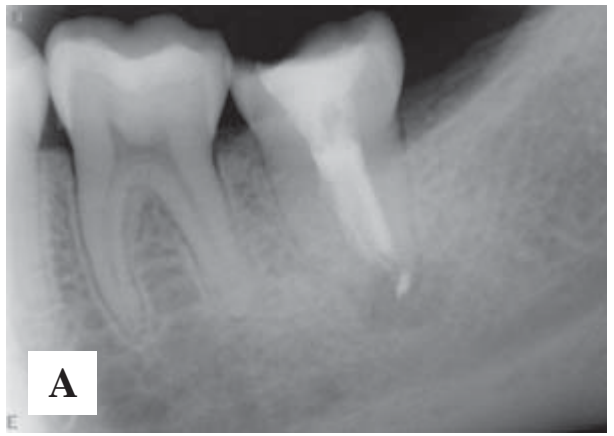

Figura 5. A: Radiografía después del tratamiento endodóntico. B: Radiografía periapical posterior al reimplante. C: Radiografía de Control, un año después del reimplante.

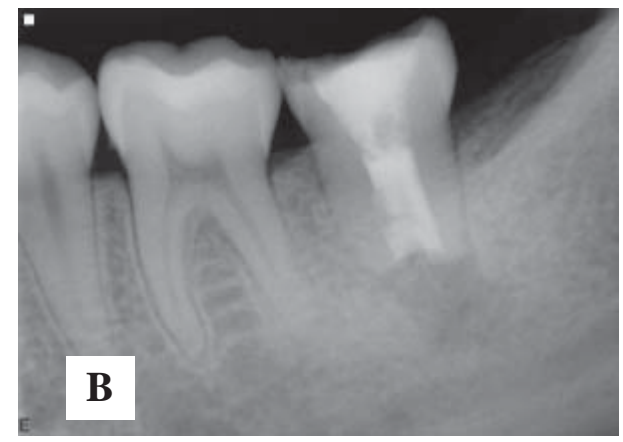

su alvéolo, el clínico debe ejercer una suave presión en las paredes vestibular y lingual. La ferulización no está indicada y debe hacerse solo cuando existan raíces cortas o falta de hueso interproximal, o cuando haya un compromiso oclusal grande, en cuyo caso se utilizará alambre ó resina (1-2 semanas) que permitan una movilidad fisiológica del diente $(17,20)$.Una ferulización prolongada puede ocasionar reabsorción o anquilosis de la raíz (11). Para todo el procedimiento es recomendado que estén siempre presentes dos operadores $(17,21)$.

La sutura debe realizarse con la menor contaminación bacteriana posible tratando de lograr la estabilización (Figura 4). Posteriormente se hará un ajuste oclusal, reducido y conservador dependiendo de la

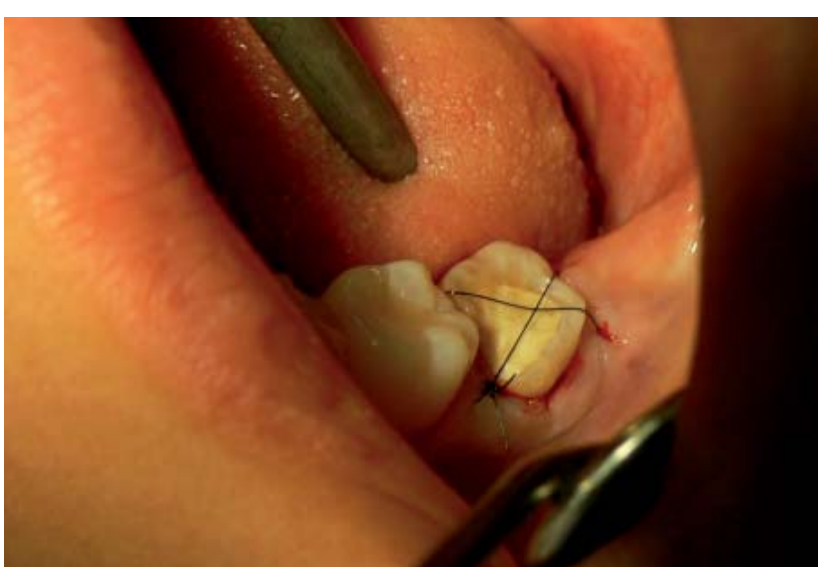

Figura4. Sutura, necesaria para conseguir la estabilización.

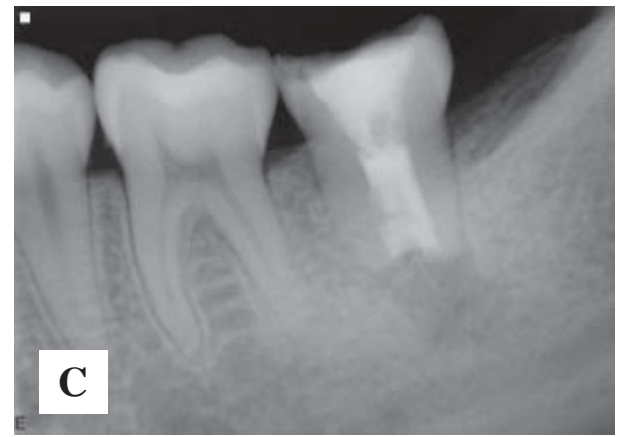


estabilización obtenida (22).

La evaluación radiográfica post-operatoria es vital para evaluar la posición de la pieza reimplantada, comparándola con la radiografía pre-operatoria (22). Los controles recomendados deberían ser por lo menos hasta un año después del procedimiento (23) (Figura 5).

A: radiografía después del tratamiento endodóntico.

B: Radiografía periapical posterior al reimplante

C: Radiografía periapical de control, seguimiento de 1 año.

Kany describe una variante del reimplante intencional por osteotomía simple para evitar las desventajas que podrían ocurrir con la técnica convencional. El procedimiento se basa en que al ser removido el diente junto con su hueso circundante, se dejarán las fibras del ligamento periodontal intactas, transfiriéndose la curación de la herida al tejido óseo (24).

\section{Principios biológicos}

Los patrones están determinados por: a) la curación del ligamento periodontal, que dependerá del tiempo operatorio y de la superficie radicular afectada, y b) la consolidación ósea, dada por la fijación de las células del ligamento periodontal (22).

\section{a) Curación del ligamento periodontal}

Una favorable curación del ligamento periodontal depende del número de células viables que se conservan en la raíz, las cuales pueden ser mecánicamente dañadas durante la extracción, o dañadas bioquímicamente debido a diversas condiciones extraorales $(2,23)$. Las células del ligamento periodontal son fácilmente dañadas bajo condiciones de estrés tales como un $\mathrm{pH}$ variable, presión osmótica alterada, deshidratación, etc. Si los dientes se extraen con un mínimo daño mecánico al ligamento periodontal y se conservan en condiciones óptimas hasta el final de la intervención quirúrgica, se debe esperar una curación exitosa. La curación óptima del ligamento periodontal se observa cuando un diente se coloca inmediatamente en su alvéolo. En esta situación, la "reinserción" se produce en 2 semanas entre los tejidos conectivos del ligamento periodontal de la superficie radicular y la pared del alvéolo. La importancia de las células progenitoras en el alvéolo no debe ser pasada por alto (22).
Otro factor importante a considerar en lo que se refiere a la curación del ligamento periodontal es la reparación de la superficie radicular dañada mecánicamente con el nuevo cemento y ligamento periodontal. La reacción inicial al trauma de la lesión es siempre una inflamación aguda $(2,18,23)$. Si no hay un estímulo adicional para mantener esta respuesta inflamatoria, la curación tendrá lugar. El tipo de curación de una superficie radicular dañada cuando un diente se reimplanta dependerá del área a ser repoblada (22).

Si el área es pequeña, las células con el potencial de formar nuevo cemento y ligamento periodontal probablemente cubrirán la superficie dañada, éste tipo de curación se denomina reabsorción superficial o curación cementaría $(19,22)$.

Sin embargo, si el área a curar es grande, las células programadas para formar hueso se adhieren en algunas zonas de la raíz, donde un proceso fisiológico de recambio óseo se llevará a cabo, la raíz se reabsorbe así como el hueso adyacente, luego el área reabsorbida se rellenará con tejido óseo, de esta manera la raíz será sustituida por hueso (22). Este proceso se ha denominado anquilosis o reabsorción por sustitución. Esta reabsorción por sustitución es irreversible y progresará hasta que se pierda el diente. La velocidad de la sustitución de la raíz dependerá de la edad del paciente, mientras más joven, más rápido $(18,19,22)$. En pacientes adultos, los dientes se pierden muy lentamente y pueden mantener la función y estética durante muchos años. En cambio, en niños pequeños antes de la pubertad, los dientes se pierden rápidamente $(22,25,26)$.

\section{b) Consolidación ósea}

El ligamento periodontal de un diente reimplantado parece ser capaz de inducir la producción de hueso. Genéticamente, las células del ligamento periodontal se pueden diferenciar en tres tipos de células: fibroblastos, cementoblastos, y osteoblastos; por lo tanto pueden generar hueso alrededor del reimplante (22). La inducción de hueso se observa como una rápida regeneración ósea y la aparición de la lámina dura alrededor del reimplante. Los injertos de hueso entre las paredes óseas y raíces son innecesarios, incluso si el espacio es amplio $(22,27)$. 


\section{Obturación retroapical}

Diversos materiales se han sugerido como materiales de obturación retroapical, incluyendo óxido de zinc-eugenol reforzado (Cavit $\left.{ }^{\circledR}\right)$, gutapercha, óxido de zinc-eugenol, resina compuesta y oro. La amalgama fue el material más usado a pesar de su microfiltración y expansión en el endurecimiento. El Super EBA ${ }^{\circledR}$, un cemento de óxido de zinc reforzado, también cuenta con bastante popularidad (28).

El Mineral Trióxido Agregado (MTA) está demostrado que sella todas las vías de comunicación entre el sistema de conductos radiculares y la superficie externa del diente, por lo tanto es el más recomendado $(28,29)$.

Los materiales biocerámicos, como el Endosequence Root Repair Material ${ }^{\circledR}$, son otra alternativa. Presentan excelentes propiedades físicas y biológicas, además son fáciles de manipular pues vienen en una pasta premezclada (29).

\section{Impacto de la tomografía en el pronóstico}

Las limitaciones de las radiografías intraorales convencionales impiden proporcionar una información suficiente para detectar la presencia de reabsorción radicular y anquilosis; o incluso evidenciar la regeneración ósea. La Tomografía Computarizada Cone Beam (TCCB) ha sido ampliamente utilizada en varios campos de la odontología, pues es capaz de producir detalles en alta resolución e imágenes en tres dimensiones de las estructuras orales $(15,30,31)$.

Kabashima y col. reportaron dos casos de reimplante intencional en dientes afectados por fractura vertical y perforación, el seguimiento con TCCB no demostró evidencia de anquilosis, más aún reveló regeneración del hueso alveolar, lo que indicó un buen pronóstico a largo plazo (30).

\section{DISCUSIÓN}

El reimplante intencional es un procedimiento considerado como un último recurso en casos donde no se puede realizar un tratamiento endodóntico quirúrgico y no quirúrgico. Debe seleccionarse solo como opción, ya que no es un tratamiento de rutina $(11,17)$. Los dentistas deben considerar los protocolos estándar, que si no son cumplidos podrían desencadenar en una reabsorción radicular y anquilosis en el plazo de uno o dos meses $(2,6,21,30)$.

Un trauma reducido durante la exodoncia será vital. Trope indica que mientras más se minimice la inflamación, el área radicular afectada será menor (18). Los procesos de reabsorción son diagnosticados dentro de los primeros dos a tres años. Sin embargo, aunque es raro, pueden ocurrir incluso después de cinco o diez años $(10,19)$. Majorana y col. siguieron 45 casos de reabsorción en trauma dental durante cinco años, registrando complicaciones y las respuestas al tratamiento. De éstos, 9 se asociaron a luxación (20\%) y 36 se asociaron a avulsión (80\%). Además, identificaron 30 casos de reabsorción radicular inflamatoria y 15 casos de anquilosis (26).

Según Tsukiboshi, una adecuada sutura es un factor importante debido a que facilitará la formación de un aparato de inserción, y sobre todo impedirá la invasión bacteriana a través del coágulo y el alvéolo (22).

Ciertamente, el porcentaje de fracaso es atribuible a la reabsorción radicular y anquilosis.Para prevenir estos factores, se han realizado estudios con variantes de la técnica y adicionando materiales que mejoren la longevidad del procedimiento $(13,22,32)$.

Kany reportó un caso por osteotomía simple, removiendo el diente junto con su hueso circundante antes de reimplantar, después de cuatro meses, la pieza estuvo funcional y asintomática (24).

Araújo y col. demostraron en raíces reimplantadas de perros, que fueron privadas de cementoblastos, que la terapia con proteínas de la matriz del esmalte (Endogaim $\left.{ }^{\circledR}\right)$ sobre la superficie radicular previamente grabada con ácido etilendiaminotetracético (EDTA) no interfirió con el proceso de curación (32). Asimismo, Demiralp y col. evaluaron los resultados del reimplante intencional en 15 dientes con periodontitis después de un grabado de las superficies radiculares con clorhidrato de tetraciclina a una concentración de $100 \mathrm{mg} / \mathrm{ml}$, durante 5 minutos. Después de seis meses, no encontraron reabsorción radicular ni anquilosis observable radiográficamente. Aunque el período de evaluación fue breve, los autores sugirieron que el reimplante intencional puede ser una alternativa a la extracción en casos hay una destrucción periodontal avanzada y ningún otro tratamiento pudiera ser considerado (33).

Al-Hezaimi y col. trataron un surco radicular en 
una paciente con un grave defecto endo-periodontal combinado, se realizó el reimplante intencional y una terapia con Emdogain ${ }^{\circledR}$. A los controles de 1 y 4 años, la paciente estuvo asintomática y la cicatrización activa fue evidente $(34,35)$. Yu y col. informaron de un caso de reimplante intencional de un molar inferior con una lesión endo-periodontal combinada, aplicando hidroxiapatita; cuatro meses después de la cirugía, se realizó una restauración coronaria, a los 15 meses de control, el diente estuvo clínicamente y radiográficamente saludable, así como estable(36).

Respecto al manejo de fracturas radiculares verticales, Hayashi y col. evaluaron veintiséis dientes con fractura radicular vertical, los cuales fueron reconstruidos con resina antes del reimplante intencional. La tasa de éxito fuedel 88,5\% a los 12 meses después de la reimplantación, el 69,2\% a los 36 meses, y el $59,3 \%$ a los 60 meses, los fracasos se debieron a una nueva fractura, recidiva de la inflamación gingival, o ambos (13).

En cuanto a la obturación retroapical, materiales más aceptados en cirugía endodóntica complementaria son el MTA, Super EBA ${ }^{\circledR}$ y los biocerámicos como el Endosequence Root Repair Material ${ }^{\circledR}$ $(28,29)$. Sin embargo, Shintani y col. realizaron un reimplante intencional en un incisivo inferior inmaduro con una lesión periapical refractaria, la obturación retroapical se hizo con una pasta de hidróxido de calcio y yodoformo, después el diente se ferulizó. Cinco años más tarde, no se encontraron anormalidades clínicas ni radiográficas y el ápice se obturó habiendo ya un puente apical (37).

Debido a que diferentes investigadores informan tasas de éxito variables, es difícil predecir el resultado del reimplante intencional. Por ejemplo Bender y Rossman evaluaron 31 casos con una tasa de éxito de $80,6 \%$ (seis fracasos registrados). Los dientes reimplantados sobrevivieron entre 1 día y 22 años. Un segundo molar inferior que fracasó después de tres semanas fue reimplantado con éxito una segunda vez, sin signos de fracaso después de 46 meses de seguimiento (10). Peer realizó 9 casos de reimplante intencional donde ilustró su viabilidad para una variedad de indicaciones. Sólo un caso de reimplante mostró evidencia de patología; su estudio sugiere que el reimplante intencional es un procedimiento fiable y predecible, y debe ser considerado más a menudo como un método de tratamiento para mantener la dentición natural (11).

De la misma forma, Aqrabawi evaluó dos casos de reimplante intencional en segundos molares infe- riores, sin encontrar radiográficamente evidencia de cambios patológicos después de 5 años de control (38).

Kaufman reportó resultados positivos en un molar superior después de un período de seguimiento de cuatro años (39), Chandra y Bhat realizaron un reimplante intencional de un segundo molar inferior para ese entonces obturado con amalgama, al control de 20 años el paciente no tuvo ningún problema con su diente (40).

Finalmente, es importante destacar que si el procedimiento es realizado utilizando una técnica correcta y se hace un adecuado seguimiento, el pronóstico será ampliamente favorable. Por lo tanto, se sugiere esta técnica en casos donde otro tratamiento conservador no sea posible realizar.

Agradecimientos: Los autores agradecen el apoyo y la experiencia del Dr. Luis Mallqui Herrada en la elaboración de este artículo de revisión.

\section{Correspondencia:}

\author{
Eric Hernán Coaguila Llerena \\ e-mail: ehernanco@gmail.com
}

\section{REFERENCIAS BIBLIOGRÁFICAS}

1. Abdurahiman VT, Jolly SJ, Khader MA. Intentional extraction and replantation: the last resort. J Indian Soc Pedod Prev Dent. 2013; 31(3):205-8.

2. Rouhani A, Javidi B, Habibi M, Jafarzadeh H. Intentional replantation: a procedure as a last resort. J Contemp Dent Pract. 2011; 12(6):486-92.

3. Emmertsen E. Replantation of extracted molars; preliminary report. Oral Surg Oral Med Oral Pathol. 1956; 9(1):115-22.

4. Dryden JA, Arens DE. Intentional replantation. A viable alternative for selected cases. Dent Clin North Am. 1994; 38(2):325-53.

5. Massler M, Tooth replantation. Dent Clin North Am. 1974; 18(2):445- 452.

6. Grossman LI. Intentional replantation of teeth. J Am Dent Assoc.1966; 72(5):1111-8.

7. Grossman LI. Intentional replantation. J Am Dent Assoc. 1980; 101(1):11-2.

8. Grossman LI. Intentional replantation of teeth: a clinical evaluation. J Am Dent Assoc. 1982; 104(5): 633-9.

9. Madison S. Intentional replantation. Oral Surg Oral Med Oral Pathol. 1986; 62(6):707-9.

10. Bender IB, Rossman LE. Intentional replantation of endodontically treated teeth. Oral Surg Oral Med Oral Pathol. 1993;76(5): 623-30.

11. Peer M. Intentional replantation - a 'last resort' treat- 
ment or a conventional treatment procedure? Nine case reports. Dent Traumatol. 2004; 20(1):48-55.

12. Selden HS. Repair of incomplete vertical root fractures in endodontically treated teeth--in vivo trials. J Endod. 1996;22(8):426-9.

13. Hayashi M, Kinomoto Y, Takeshige F, Ebisu S. Prognosis of intentional replantationof vertically fractured roots reconstructed with dentin-bonded resin. J Endod. 2004;30(3):145-8.

14. Wang Z,Heffernan M, Vann WF Jr. Management of a complicated crown-rootfracture in a young permanent incisor using intentional replantation. Dent Traumatol. 2008;24(1):100-3.

15. Liang $X$, Jacobs $R$, Hassan B, et al. A comparative evaluation of Cone Beam Computed Tomography (CBCT) and Multi-Slice CT (MSCT) Part I. On subjective image quality. Eur J Radiol. 2010; 75(2): 2659.

16. Peñarrocha M, García B, Martí E, Palop M, von Arx T. Intentional replantationfor the management of maxillary sinusitis. Int Endod J. 2007; 40(11):891-9.

17. Cotter MR, Panzarino J. Intentional replantation: a case report. J Endod. 2006; 32(6):579-82.

18. Trope M. Root resorption due to dental trauma. Endod Topics 2002; 1:79-100.

19. Andreasen JO, Hørting-Hansen E. Replantation of teeth: Radiographic and clinical study of 110 human teeth reimplanted after accidental loss. Acta Odontol Scand. 1966: 24(3): 263-286.

20. Herrera H, Leonardo MR, Herrera H, Miralda L, Bezerra da Silva RA. Intentional replantation of a mandibular molar: case report and 14-year follow-up. Oral Surg Oral Med Oral Pathol Oral Radiol Endod. 2006; 102(4):85-7.

21. WardJ. Intentional replantation of a lower premolar. Aust Endod J. 2004 ;30(3):99-102.

22. Tsukiboshi M. Autotransplantation of teeth: requirements for predictable success. Dent Traumatol.2002;18(4):157-80.

23. Guy SC, Goerig AC. Intentional replantation: technique and rationale.Quintessence Int Dent Dig. 1984; 15(6):595-603.

24. Kany FM. Single-tooth osteotomy for intention replantation. J Endod. 2002; 28(5):408-10.

25. Cvek M, Lindvall A. External root resorption following bleaching of pulpless teeth with oxygen peroxide. Endod Dent Traumatol 1985;1(2):56-60.

26. Majorana A, Bardellini E, Conti G, Keller E, Pasini S. Root resorption in dental trauma: 45 cases followed for 5 years. Dent Traumatol. 2003; 19(5):262-5.

27. Andreasen JO. Interrelation between alveolar bone and periodontal ligament repair after replantation of mature permanent incisors in monkeys. J Periodontal Res 1981;16(2):228-35.

28. Aqrabawi J. Sealing ability of amalgam, super EBA cement, and MTA when used as retrograde filling materials. Br Dent J. 2000;188(5):266-8.

29. Hansen S, Marshall G, Sedgley C. Comparison of intracanal Endo sequence Root Repair Material and ProRoot MTA to induce $\mathrm{pH}$ changes in simulated root resorption defects over 4 weeks in matched pairs of human teeth. J Endod. 2011;37(4):502-506.

30. Kabashima H, Mizobe K, Sakai T, Nakamuta H, Kurita K, Terada Y. The usefulness of three-dimensional imaging for prognostication in cases of intentional tooth replantation. J Oral Sci. 2012; 54(4):355-8.

31. Nair MK, Nair UP. Digital and advanced imaging in endodontics: a review. J Endod. 2007; 33(1):1-6.

32. Araújo M, Hayacibara R, Sonohara M, Cardaropoli G, Lindhe J. Effect of enamel matrix proteins (Emdogain $\left.{ }^{\circledR}\right)$ on healing after re-implantation of "periodontally compromised" roots. An experimental study in the dog. J Clin Periodontol. 2003; 30(10):855-61.

33. Demiralp B, Nohutçu RM, Tepe DI, Eratalay K. Intentional replantation for periodontally involved hopeless teeth. Dent Traumatol. 2003;19(1):45-51.

34. Al-Hezaimi K, Naghshbandi J, Simon JH, Oglesby S, Rotstein I. Successful treatment of a radicular groove byintentional replantationand Emdogain $₫$ therapy. Dent Traumatol. 2004;20(4):226-8.

35. Al-Hezaimi K, Naghshbandi J, Simon JH, Rotstein I. Successful treatment of a radicular groove byintentional replantation and Emdogain ${ }^{\circledR}$ therapy: four years follow-up. Oral Surg Oral Med Oral Pathol Oral Radiol Endod. 2009; 107(3):82-5.

36. Yu L, Xu B, Wu B. Treatment of combined endodontic-periodontic lesions by intentional replantation and application of hydroxyapatites. Dent Traumatol. 2003; 19(1):60-3.

37. Shintani S, Tsuji M, Toyosawa S, Ooshima T. Intentional replantation of an immature permanent lower incisor because of a refractory periapical lesion: case report and 5-year follow-up. Int J Paediatr Dent. 2004; 14(3):218-22.

38. Aqrabawi J. Five-year follow-up of successful intentional replantation. Dent Update. 1999; 26(9):388-90.

39. Kaufman AY. Intentionalreplantationof a maxillary molar.:A 4-year follow-up. Oral Surg Oral Med Oral Pathol.1982; 54(6):686-8.

40. Chandra RV,Bhat KM. Twenty-year follow-up of an unconventional intentional replantation. J Can Dent Assoc. 2006; 72(7):639-42.

Recibido : 08/12/2014

Aceptado: 15/04/2015 